\title{
Effect of 3-month $\alpha$-lipoic acid treatment on sural nerve conduction velocity and amplitude in patients with diabetic neuropathy: a pilot study
}

Athanasia Papazafeiropoulou, Eleni Xourgia, Styliani Papantoniou, Aikaterini Trikkalinou, Andreas Melidonis

$1^{\text {st }}$ Department of Internal Medicine and Diabetes Center, General Hospital of Piraeus "Tzaneio", Piraeus, Greece

Submitted: 27 April 2019

Accepted: 22 June 2019

Arch Med Sci Atheroscler Dis 2019; 4: e141-e143 DOI: https://doi.org/10.5114/amsad.2019.86750 Copyright (C) 2019 Termedia \& Banach

Here we present our original research on the effects of $\alpha$-lipoic acid, an emerging treatment option for the management of diabetic neuropathy, on nerve conduction parameters, an indirect marker of nerve functional integrity.

Diabetic neuropathy (DN) is a common complication of type 2 diabetes (T2D) with a major impact on the patient's quality of life. Despite therapeutic advances, there are no agents targeting the main pathogenetic mechanisms of DN [1]. One of these is oxidative stress [2]. A-lipoic acid (ALA) seems to delay or reverse peripheral diabetic neuropathy through its multiple antioxidant properties [3]. Therefore, the aim of our study was to explore the effect of ALA treatment on the sural nerve conduction velocity (SNCV) and amplitude (SNAP) of T2D patients with DN.

Our sample consisted of 32 consecutive T2D patients (12 male), with mean age ( \pm standard deviation) $67.9 \pm 8.6$ years, glycated hemoglobin $\left(\mathrm{HbA}_{1 \mathrm{c}}\right) 7.1 \pm 1.0 \%$, body mass index $30.7 \pm 11.2 \mathrm{~kg} / \mathrm{m}^{2}$ and T2D duration $14.1 \pm 5.3$ years, attending the diabetes outpatient clinic of our hospital between September 2018 and February 2019. 72.7\% of study patients were on oral antidiabetic medication and $26.5 \%$ on insulin therapy. Diagnosis of DN was based on the presence of at least one neuropathic symptom (burning, shooting pain, paraesthesia, muscle cramps or allodynia) in the lower extremities, and previous treatment for DN for $\geq 3$ months before enrollment in the study [1]. Patients with causes of neuropathy other than diabetes (such as chronic alcohol misuse, vitamin $B_{12}$ deficiency, drug-induced neuropathy), truncal neuropathy or severe neurological diseases (such as Parkinson's disease and multiple sclerosis) and severe renal disease defined as estimated glomerular filtration rate (eGFR) $<30 \mathrm{ml} /$ $\mathrm{min} / 1.73 \mathrm{~m}^{2}$ were excluded from the study. Regarding the other microand macrovascular diabetic complications, $14.3 \%$ of study patients had retinopathy, $28.6 \%$ chronic kidney disease, $18.2 \%$ coronary artery disease, and $47.6 \%$ peripheral arterial disease.

Participants were prescribed $600 \mathrm{mg} /$ day ALA (Combinerv), to be administered orally, for 3 months, and were advised not to discontinue any other medication for DN, antidiabetic drugs, or substances used for managing arterial hypertension or dyslipidemia during the study. All study patients underwent a complete clinical examination and measurement of SNCV and SNAP using the NCstat DPNCheck test (NeuroMetrix, Inc.,

\author{
Corresponding author: \\ Dr. Eleni Xourgia \\ $1^{\text {st }}$ Department \\ of Internal Medicine \\ and Diabetes Center \\ General Hospital \\ of Piraeus "Tzaneio" \\ Piraeus, Greece \\ E-mail: elena.xourgia@gmail. \\ com
}


Waltham, MA) at study entry and after 3 months of ALA treatment. The NC-stat DPNCheck test has been assessed as both reliable and valid, and has the potential to be suitable for clinical applications [4]. Neuropathy was assessed using the Neuropathy Symptom Score (NSS) and the Neuropathy Disability Score (NDS) questionnaires at study entry and 3 months later [1].

The study was approved by the Ethics Committee of General Hospital of Piraeus "Tzaneio", and was conducted in accordance with the Declaration of Helsinki. Written informed consent was obtained from all participants.

Data were analyzed using SPSS software, version 22.0 (IBM, Armonk, NY, USA) for Windows. Continuous variables are presented as mean \pm SD. Nominal variables are presented as $n(\%)$ prevalence or frequencies. Student's $t$-test was used to compare total scores/measurements between the baseline and $2^{\text {nd }}$ visit. All tests were two-sided with $\alpha=0.05$. A $p$-value $\leq 0.05$ was considered statistically significant.

At study entry, mean SNCV and SNAP were $45.5 \pm 17.6 \mathrm{~m} / \mathrm{s}$ and $3.3 \pm 1.5 \mu \mathrm{V}$, respectively. After 2 months of $\alpha$-lipoic acid treatment, SNCV and SNAP were $41.1 \pm 16.0 \mathrm{~m} / \mathrm{s}$ and $4.1 \pm 1.6 \mu \mathrm{V}$, respectively. There were no statistically significant differences between SNCV and SNAP at study entry and after 3 months of ALA treatment. At study entry, NSS and NDS scores were $8.6 \pm 2.7$ and 5.4 \pm 1.2 , respectively. After 3 months of ALA treatment, NSS and NDS scores were $5.6 \pm 2.1$ and 5.5 \pm 1.1 , respectively. Only the observed decrease in NSS score was statistically significant $(p=0.05)$. No side effects were detected at the end of the study.

The results of the present study showed no effect of 3-month ALA treatment on SNCV and SNAP. In fact, there is no current evidence regarding an effective therapy for enhancing peripheral nerve function in patients with DN although a recent review showed that several treatments, including $A L A$, are effective in improving DN symptoms $[4,5]$. In accordance with the above, a multicenter study for the evaluation of the efficacy and safety of ALA over 4 years in mild-to-moderate diabetic distal symmetric sensorimotor polyneuropathy showed that treatment with ALA did not influence neurophysiologic tests, but showed clinically meaningful improvement of neuropathic impairments [6]. However, a study in 50 patients with T2D and a deficit in both motor and sensory nerve conduction showed that after 4 months of treatment with ALA, patients significantly improved their electroneurographic parameters and their perception of pain [7]. The best improvements were observed in sensory nerve conduction [7]. Another study, in 30 patients with T2D with symptoms of DN for $\geq 6$ months, showed that the combination of ALA with methylcobalamin and pregabalin resulted in improvement of nerve function [8]. In addition, a study in 24 male Wistar rats with DN showed that 2 weeks of treatment with ALA significantly increased nerve conduction velocity and amplitude [9]. In contrast, another study using diabetic rats showed that ALA treatment had no effect on sensory tests or on antioxidant activity [10].

It is obvious that the existing literature data on the effect of ALA on peripheral nerve function are still limited and controversial, with some studies showing a beneficial effect and others a neutral effect. At this point, it must be mentioned that our study has some limitations. First of all, we did not have a control group and patient's compliance with the study agent was not assessed. Secondly, the sample was small, and the duration of the study short. Therefore, our results should be interpreted with reservation.

In conclusion, ALA treatment failed to improve SNCV and SNAP in T2D patients with DN. Interestingly, $\alpha$-lipoic acid treatment induced an improvement only in NSS score. Longer patient follow-up might be necessary to show the favorable effect of ALA on DN.

\section{Conflict of interest}

The authors declare no conflict of interest.

\section{References}

1. Boulton AJ, Vinik AI, Arezzo JC, et al. Diabetic neuropathies: a statement by the American Diabetes Association. Diabetes Care 2005; 28: 956-62.

2. Brownlee $M$. The pathobiology of diabetic complications: a unifying mechanism. Diabetes 2005; 54: 1615-25.

3. Nagamatsu M, Nickander KK, Schmelzer JD, et al. Lipoic acid improves nerve blood flow, reduces oxidative stress and improves distal nerve conduction in experimental diabetic neuropathy. Diabetes Care 1995; 18: 1160-7.

4. Chatzikosma G, Pafili K, Demetriou M, Vadikolias K, Maltezos E, Papanas N. Evaluation of sural nerve automated nerve conduction study in the diagnosis of peripheral neuropathy in patients with type 2 diabetes mellitus. Arch Med Sci 2016; 12: 390-3.

5. Lee CC, Perkins BA, Kayaniyil S, et al. Peripheral neuropathy and nerve dysfunction in individuals at high risk for type 2 diabetes: the PROMISE cohort. Diabetes Care 2015; 38: 793-800.

6. Ziegler D, Low PA, Litchy WJ, et al. Efficacy and safety of antioxidant treatment with alpha-lipoic acid over 4 years in diabetic polyneuropathy: the NATHAN 1 trial. Diabetes Care 2011; 34: 2054-60.

7. Bertolotto F, Massone A. Combination of alpha lipoic acid and superoxide dismutase leads to physiological and symptomatic improvements in diabetic neuropathy. Drugs R D 2012; 12: 29-34.

8. Vasudevan D, Naik MM, Mukaddam QI. Efficacy and safety of methylcobalamin, alpha lipoic acid and pregabalin combination versus pregabalin monotherapy in improving pain and nerve conduction velocity in type 2 
diabetes associated impaired peripheral neuropathic condition. [MAINTAIN]: results of a pilot study. Ann Indian Acad Neurol 2014; 17: 19-24.

9. Seyit DA, Degirmenci E, Oguzhanoglu A. Evaluation of electrophysiological effects of melatonin and alpha lipoic acid in rats with streptozotocine induced diabetic neuropathy. Exp Clin Endocrinol Diabetes 2016; 124: 300-6.

10. Lee KA, Lee NY, Park TS, Jin HY. Comparison of periph eral nerve protection between insulin-based glucose control and alpha lipoic acid (ALA) in the streptozotocin (STZ)-induced diabetic rat. Endocrine 2018; 61: 58-67. 